\title{
COMPOSITION OF MAXIMAL OPERATORS
}

\author{
Menita Carozza and Antonia Passarelli di Napoli
}

Abstract

Consider the Hardy-Littlewood maximal operator

$$
M f(x)=\sup _{Q \ni x} \frac{1}{|Q|} \int_{Q}|f(y)| d y .
$$

It is known that $M$ applied to $f$ twice is pointwise comparable to the maximal operator $M_{L \log L} f$, defined by replacing the mean value of $|f|$ over the cube $Q$ by the $L \log L$-mean, namely

$$
M_{L \log L} f(x)=\sup _{x \in Q} \frac{1}{|Q|} \int_{Q}|f(y)| \log \left(e+\frac{|f|}{|f|_{Q}}\right)(y) d y,
$$

where $|f|_{Q}=\frac{1}{|Q|} \int_{Q}|f|$ (see $[\mathbf{L}],[\mathbf{L N}],[\mathbf{P}]$ ).

In this paper we prove that, more generally, if $\Phi(t)$ and $\Psi(t)$ are two Young functions, there exists a third function $\Theta(t)$, whose explicit form is given as a function of $\Phi(t)$ and $\Psi(t)$, such that the composition $M_{\Psi} \circ M_{\Phi}$ is pointwise comparable to $M_{\Theta}$. Through the paper, given an Orlicz function $A(t)$, by $M_{A} f$ we mean

$$
M_{A} f(x)=\sup _{Q \ni x}\|f\|_{A, Q}
$$

where $\|f\|_{A, Q}=\inf \left\{\lambda>0: \frac{1}{|Q|} \int_{Q} A\left(\frac{|f|}{\lambda}\right)(x) d x \leq 1\right\}$.

\section{Introduction.}

Let $f \in L_{\text {loc }}^{1}\left(\mathbb{R}^{n}\right)$, the Hardy-Littlewood maximal operator $M f$ of $f$ is defined by

$$
M f(x)=\sup _{Q \ni x} \frac{1}{|Q|} \int_{Q}|f(y)| d y .
$$

This work has been supported by M.U.R.S.T. (40\%). 
A well-known result of Coifman and Rochberg, (see [CR], [T]), states that if $M f<\infty$ a.e. and if $\delta \in(0,1)$, then $(M f)^{\delta} \in A_{1}$, where $A_{1}$ is the Muckenhoupt class of the non negative weights $w$ such that

$$
A_{1}(w)=\sup _{Q} \frac{f_{Q} w}{\operatorname{essinf}_{Q} w}<\infty,
$$

where $f_{Q} w$ stands for the average of $w$ over $Q$ and the supremum being taken over all cubes $Q$ of $\mathbb{R}^{n}$.

Setting

$$
M_{r} f=\sup _{Q \ni x}\left(f_{Q}|f|^{r}\right)^{\frac{1}{r}} \quad r>1,
$$

from the mentioned result, with $\delta=\frac{1}{r}$, it follows that $M \circ M_{r} \sim M_{r}$. This means that there exists a constant $c$, such that

$$
M_{r} f(x) \leq M\left(M_{r} f(x)\right) \leq c M_{r} f(x) \text { a.e. in } \mathbb{R}^{n} .
$$

For $r=1$ the situation is different, namely we have that $M \circ M \sim$ $M_{L \log L}$, i.e.

$$
c_{1} M_{L \log L} f(x) \leq M(M f(x)) \leq c_{2} M_{L \log L} f(x) \quad \text { a.e. in } \quad \mathbb{R}^{n}
$$

(see $[\mathbf{L}],[\mathbf{L N}],[\mathbf{P}]$ ), and this corresponds to Stein's result, i.e. for $f$ supported in a cube $Q$

$$
f \in L \log L(Q) \Longleftrightarrow M f \in L^{1}(Q)
$$

(see [S]). The maximal operator $M_{L \log L} f$ is defined by replacing the mean value of $|f|$ over the cube $Q$ by the $L \log L$-mean, namely

$$
M_{L \log L} f(x)=\sup _{x \in Q} \frac{1}{|Q|} \int_{Q}|f(y)| \log \left(e+\frac{|f|}{|f|_{Q}}\right)(y) d y
$$

where $|f|_{Q}=\frac{1}{|Q|} \int_{Q}|f|$.

The previous results justify the introduction of a maximal operator in an Orlicz space such as $L \log L$.

More precisely, let $\Omega$ be a cube of $\mathbb{R}^{n}$. A continuosly increasing function on $[0, \infty]$, say $\Psi:[0, \infty] \rightarrow[0, \infty]$ such that $\Psi(0)=0, \Psi(1)=1$ and $\Psi(\infty)=\infty$, will be referred to as an Orlicz function. 
The generalized Orlicz space denoted by $L^{\Psi}(\Omega)$ consists of all functions $g: \Omega \subseteq \mathbb{R}^{n} \rightarrow \mathbb{R}$ such that

$$
\int_{\Omega} \Psi\left(\frac{|g|}{\lambda}\right)(x) d x<\infty
$$

for some $\lambda>0$.

Let us define the $\Psi$-average of $g$ over a cube $Q$ contained in $\Omega$ by

$$
\|g\|_{\Psi, Q}=\inf \left\{\lambda>0: f_{Q} \Psi\left(\frac{|g|}{\lambda}\right)(x) d x \leq 1\right\} .
$$

When $\Psi(t)$ is a Young function, i.e. a convex Orlicz function, the quantity

$$
\|g\|_{\Psi}=\inf \left\{\lambda>0: \int_{\Omega} \Psi\left(\frac{|g|}{\lambda}\right)(x) d x \leq 1\right\}
$$

is the well known Luxemburg norm in the space $L^{\Psi}(\Omega)$ (see $[\mathbf{K R}],[\mathbf{R R}]$ ).

If $f \in L^{\Psi}\left(\mathbb{R}^{n}\right)$, the maximal function of $f$ with respect to $\Psi$ is defined by setting

$$
M_{\Psi} f(x)=\sup _{x \in Q}\|f\|_{\Psi, Q}
$$

where the supremum is taken over all cubes $Q$ of $\mathbb{R}^{n}$ containing $x$ with sides parallel to the coordinate axes.

Let us remark that if we choose $\Psi(t)=t \log (e+t)$, the maximal operator $M_{\Psi} f$ defined by (1.3) is equivalent to the $M_{L} \log L$ operator defined by (1.1) (see [IS]).

In this paper we generalize the mentioned results: namely, given two Young functions $\Phi(t)$ and $\Psi(t)$, we get a third Young function $\Theta(t)$, such that the composition, $M_{\Psi} \circ M_{\Phi}$, between $M_{\Phi}$ and $M_{\Psi}$ is equivalent to the operator $M_{\Theta}$.

As an application, we reobtain, in a simple way, the Herz type inequality for the nonincreasing rearrangement of the maximal operator in $L \log L($ see $[\mathbf{B}])$.

Moreover, we obtain a pointwise estimate for the maximal function of the jacobian of a function $f$ such that $|D f|^{n}$ belongs to $L^{1}$.

\section{The main result.}

Let $\Omega$ be a cube of $\mathbb{R}^{n}$ and set

$$
\overline{\mathcal{M}}_{\Phi} f(x)=\sup _{x \in Q \subseteq \Omega}\|f\|_{\Phi, Q} .
$$

First, let us prove a result which will be useful in the following. 
Theorem 1. Let $\Psi(t)$ be an Orlicz function and $\Phi(t)$ be a Young one. For

$$
\Theta(t)=\int_{0}^{t} \Psi^{\prime}(s) \Phi\left(\frac{t}{s}\right) d s
$$

there exist two positive constants $c_{1}, c_{2}$ such that

$$
c_{1}\left\|\overline{\mathcal{M}}_{\Phi} f\right\|_{\Psi, \Omega} \leq\|f\|_{\Theta, \Omega} \leq c_{2}\left\|\overline{\mathcal{M}}_{\Phi} f\right\|_{\Psi, \Omega}
$$

for every $f \in L^{\Theta}(\Omega)$.

Proof: In order to prove that

$$
\left\|\overline{\mathcal{M}}_{\Phi} f\right\|_{\Psi, \Omega} \leq c\|f\|_{\Theta, \Omega}
$$

we use the following equality:

$$
\int_{\Omega} \Psi\left(\frac{\overline{\mathcal{M}}_{\Phi} f(x)}{\lambda}\right) d x=\int_{0}^{\infty} \Psi^{\prime}(t)\left|\left\{x \in \Omega: \overline{\mathcal{M}}_{\Phi} f(x)>t \lambda\right\}\right| d t .
$$

Let us set

$$
E_{t \lambda}=\left\{x \in \Omega: \overline{\mathcal{M}}_{\Phi} f(x)>t \lambda\right\}
$$

Thanks to Proposition 4.1 in $[\mathbf{B P}]$, we can consider a sequence of cubes $\left\{Q_{k}\right\}$ such that

$$
E_{t \lambda}=\cup_{k} Q_{k} \quad \text { and } \quad \int_{Q_{k}} \Phi\left(\frac{|f|}{\lambda t}\right)(x) d x>\left|Q_{k}\right| .
$$

Now, we observe that

$$
\begin{aligned}
& \left|Q_{k}\right|<\int_{Q_{k}} \Phi\left(\frac{|f|}{\lambda t}\right)(x) d x \\
= & \int_{\left\{x \in \Omega:|f|>\frac{\lambda t}{2}\right\} \cap Q_{k}} \Phi\left(\frac{|f|}{\lambda t}\right)(x) d x+\int_{\left\{x \in \Omega:|f| \leq \frac{\lambda t}{2}\right\} \cap Q_{k}} \Phi\left(\frac{|f|}{\lambda t}\right)(x) d x \\
& \leq \int_{\left\{x \in \Omega:|f|>\frac{\lambda t}{2}\right\} \cap Q_{k}} \Phi\left(\frac{|f|}{\lambda t}\right)(x) d x+\Phi\left(\frac{1}{2}\right)\left|Q_{k}\right| .
\end{aligned}
$$

Without loss of generality, we may assume $\Phi\left(\frac{1}{2}\right)<1$, then we have

$$
\begin{aligned}
\left|Q_{k}\right| & <c \int_{\left\{x \in \Omega:|f|>\frac{\lambda t}{2}\right\} \cap Q_{k}} \Phi\left(\frac{|f|}{\lambda t}\right)(x) d x \\
& <c \int_{\left\{x \in \Omega:|f|>\frac{\lambda t}{2}\right\} \cap Q_{k}} \Phi\left(\frac{2|f|}{\lambda t}\right)(x) d x
\end{aligned}
$$


by monotonicity of $\Phi$.

We get

$$
\left|E_{t \lambda}\right| \leq c \int_{\left\{x \in \Omega:|f|>\frac{\lambda t}{2}\right\} \cap E_{t \lambda}} \Phi\left(\frac{2|f|}{\lambda t}\right)(x) d x .
$$

After that, we obtain

$$
\begin{aligned}
\int_{\Omega} \Psi\left(\frac{\overline{\mathcal{M}}_{\Phi} f(x)}{\lambda}\right) d x & \leq c \int_{0}^{\infty} \Psi^{\prime}(t) \int_{\left\{x \in \Omega:|f|>\frac{\lambda t}{2}\right\}} \Phi\left(\frac{2|f|}{\lambda t}\right)(x) d x d t \\
& =c \int_{\Omega} \int_{0}^{\frac{2|f|}{\lambda}} \Psi^{\prime}(t) \Phi\left(\frac{2|f|}{\lambda t}\right)(x) d t d x \\
& =c \int_{\Omega} \Theta\left(\frac{2|f|}{\lambda}(x)\right) d x .
\end{aligned}
$$

By estimate above, we have (2.2). Now, we have to prove that

$$
\|f\|_{\Theta, \Omega} \leq c|| \overline{\mathcal{M}}_{\Phi} f \|_{\Psi, \Omega} .
$$

By Calderon-Zygmund lemma, we may cover $E_{t \lambda}=\left\{x \in \Omega: \overline{\mathcal{M}}_{\Phi} f(x)>\right.$ $t \lambda\}$ by a sequence of nonoverlapping cubes $Q_{k}$, each having the property

$$
2^{-n}\left|Q_{k}\right| \leq\left|Q_{k} \cap E_{t \lambda}\right|<\left|Q_{k}\right|
$$

and such that

$$
2^{n}\left|E_{t \lambda}\right| \geq \sum\left|Q_{k}\right| \geq \sum \int_{Q_{k}} \Phi\left(\frac{|f|}{\lambda t}\right) d x \geq \int_{E_{t \lambda}} \Phi\left(\frac{|f|}{\lambda t}\right) d x .
$$

We have that

$$
\int_{\Omega} \Psi\left(\frac{\overline{\mathcal{M}}_{\Phi} f(x)}{\lambda}\right) d x \geq \tilde{c} \int_{\Omega} \Theta\left(\frac{|f|}{\lambda}(x)\right) d x
$$

In fact

$$
\begin{aligned}
\int_{\Omega} \Psi\left(\frac{\overline{\mathcal{M}}_{\Phi} f(x)}{\lambda}\right) d x & =\int_{0}^{\infty} \Psi^{\prime}(t)\left|\left\{x \in \Omega: \overline{\mathcal{M}}_{\Phi} f(x)>t \lambda\right\}\right| d t \\
& \geq c \int_{0}^{\infty} \Psi^{\prime}(t) \int_{E_{t \lambda}} \Phi\left(\frac{|f|}{t \lambda}\right) d x d t \\
& =c \int_{\Omega} \int_{0}^{\frac{\overline{\mathcal{M}}_{\Phi}(f)}{\lambda}} \Psi^{\prime}(t) \Phi\left(\frac{|f|}{t \lambda}\right) d t d x \\
& \geq c \int_{\Omega} \int_{0}^{\frac{f(x)}{\lambda}} \Psi^{\prime}(t) \Phi\left(\frac{|f|}{t \lambda}\right) d t d x
\end{aligned}
$$


since $\Phi(t)$ is convex.

Finally, we get

$$
\int_{\Omega} \Psi\left(\frac{\overline{\mathcal{M}}_{\Phi} f(x)}{\lambda}\right) d x \geq c \int_{\Omega} \Theta\left(\frac{|f|}{\lambda}\right) d x
$$

which implies (2.4), then the theorem is proved

Remark 1. Theorem 1 with $\Phi$ and $\Psi$ both Young functions, is proved in $[\mathbf{B P}]$.

Moreover, in the particular case $\Phi(t)=t$ and $\Psi(t)$ any Orlicz function, Theorem 1 gives Proposition 3.1 of [GIM].

Using the previous result, we develop a useful estimate for the composition $M_{\Psi} \circ M_{\Phi}$, where $\Phi$ and $\Psi$ are Young functions.

Theorem 2. Let $\Psi(t)$ and $\Phi(t)$ be two Young functions. For

$$
\Theta(t)=\int_{0}^{t} \Psi^{\prime}(s) \Phi\left(\frac{t}{s}\right) d s
$$

there exist two positive constants, $c_{1}$ and $c_{2}$, such that for every $f \in$ $L_{\text {loc }}^{\Theta}\left(\mathbb{R}^{n}\right)$ we have

$$
c_{1} M_{\Theta} f(x) \leq M_{\Psi}\left(M_{\Phi} f(x)\right) \leq c_{2} M_{\Theta} f(x)
$$

almost everywhere.

Proof: Let us fix $x \in \mathbb{R}^{n}$ and a cube $Q$ containing $x$. Put $f=f_{1}+f_{2}$ with $f_{1}=f \chi_{3 Q}$, we have, by triangle inequality of the Luxemburg norm \|\|$_{\Psi}$,

$$
\left\|M_{\Phi} f\right\|_{\Psi, Q} \leq\left\|M_{\Phi} f_{1}\right\|_{\Psi, Q}+\left\|M_{\Phi} f_{2}\right\|_{\Psi, Q}=I+I I .
$$

In order to estimate $I$, consider

$$
\overline{\mathcal{M}}_{\Phi} f(x)=\sup \left\{\|f\|_{\Phi, \bar{Q}}: x \in \bar{Q}, \bar{Q} \subseteq 3 Q\right\}
$$

and we observe that there exists a constant $c(n)$ such that

$$
M_{\Phi} f_{1}(x) \leq c(n) \overline{\mathcal{M}}_{\Phi} f_{1}(x)
$$


Namely, for every cube $\tilde{Q} \subseteq \mathbb{R}^{n}, \tilde{Q} \ni x, \tilde{Q} \cap \mathcal{C}(3 Q) \neq \emptyset$ the following inequality holds

$$
f_{\tilde{Q}} \Phi\left(\left|f_{1}\right|\right)=\frac{1}{|\tilde{Q}|} \int_{\tilde{Q} \cap 3 Q} \Phi\left(\left|f_{1}\right|\right) \leq 3^{n} f_{3 Q} \Phi\left(\left|f_{1}\right|\right)
$$

and then, if $\lambda>0$ is such that

$$
f_{3 Q} \Phi\left(\frac{\left|f_{1}\right|}{\lambda}\right) \leq 1
$$

we have

$$
\frac{1}{3^{n}} \int_{\tilde{Q}} \Phi\left(\frac{\left|f_{1}\right|}{\lambda}\right) \leq 1
$$

By convexity of $\Phi$, we get

$$
f_{\tilde{Q}} \Phi\left(\frac{\left|f_{1}\right|}{3^{n} \lambda}\right) \leq 1
$$

and this implies

$$
\left\|f_{1}\right\|_{\Phi, \tilde{Q}} \leq 3^{n}\left\|f_{1}\right\|_{\Phi, 3 Q} .
$$

Note that (2.9) is trivial if $\tilde{Q} \subseteq 3 Q$.

Observing that $\left\|f_{1}\right\|_{\Phi, 3 Q} \leq \overline{\mathcal{M}}_{\Phi} f_{1}(x)$ and taking the supremum over all cubes $\tilde{Q}$ of $\mathbb{R}^{n}$ containing $x$ on the left hand side of (2.9), we have (2.8). By formulas (2.8) and (2.2), applied with $\overline{\mathcal{M}}$ and $\Omega=3 Q$, we deduce

$$
I=\left\|M_{\Phi} f_{1}\right\|_{\Psi, Q} \leq C\|f\|_{\Theta, Q} .
$$

To estimate $I I$ it suffices to observe that

$$
M_{\Phi} f_{2}(y) \leq C \inf _{Q} M_{\Phi} f_{2} \quad \forall y \in Q .
$$

In fact, let us fix a point $y \in Q$ and a cube $\bar{Q} \ni y$ such that $\bar{Q} \cap \mathcal{C}(3 Q) \neq \emptyset$; the cube $3 \bar{Q}$ contains every point $x \in Q$. Reasoning as before, we obtain

$$
\left\|f_{2}\right\|_{\Phi, \bar{Q}} \leq 3^{n}\left\|f_{2}\right\|_{\Phi, 3 \bar{Q}} \leq C M_{\Phi} f_{2}(x)
$$

and so (2.10). Now we observe that there are positive constants $c_{1}, c_{2}, t_{0}$, depending on $\Phi$ and $\Psi$, such that $\Phi\left(c_{1} t\right) \leq c_{2} \Theta(t)$, for $t \geq t_{0}$. Namely,

$$
\begin{aligned}
\Theta(t) & =\int_{0}^{t} \Psi^{\prime}(s) \Phi\left(\frac{t}{s}\right) d s \\
& \geq \int_{0}^{t} \Psi(s) \Phi^{\prime}\left(\frac{t}{s}\right) \frac{t}{s^{2}} d s \\
& \geq c_{0} \int_{t_{0}}^{t} \Phi^{\prime}\left(\frac{t}{s}\right) \frac{t}{s^{2}} d s \\
& =c_{0} \int_{1}^{\frac{t}{t_{0}}} \Phi^{\prime}(\sigma) d \sigma=c_{2}^{-1} \Phi\left(\frac{t}{t_{0}}\right)
\end{aligned}
$$


and then there exists a positive constant $c_{3}$ such that $M_{\Phi} f(x) \leq c_{3} M_{\Theta} f(x)$ a.e. .

By (2.7), (2.9) and (2.10), we conclude that

$$
\left\|M_{\Phi} f\right\|_{\Psi, Q} \leq C_{1}\|f\|_{\Theta, Q}+C_{2} M_{\Theta} f(x) .
$$

Taking the supremum over the cubes $Q$ containing $x$ in (2.11), we get

$$
M_{\Psi}\left(M_{\Phi} f(x)\right) \leq c_{2} M_{\Theta} f(x) \quad \text { a.e. . }
$$

On the other hand, formula (2.4) implies that

$$
M_{\Psi}\left(M_{\Phi} f(x)\right) \geq c_{1} M_{\Theta} f(x) \quad \text { a.e. }
$$

Formulas (2.12) and (2.13) give the thesis.

Let us give some example of such compositions.

Example 1. Let us consider

$$
\begin{aligned}
& \Psi(t)=t^{p} \\
& \Phi(t)=\left\{\begin{array}{ll}
1 & t \leq 1 \\
t^{q} & t>1,
\end{array} \quad p, q \geq 1\right.
\end{aligned}
$$

recalling that

$$
M_{r} f(x)=\sup _{x \in Q}\left(f_{Q} f^{r}\right)^{\frac{1}{r}}
$$

we have

$$
\begin{aligned}
& M_{p} f(x)=M_{\Psi} f(x) \\
& M_{q} f(x)=M_{\Phi} f(x) .
\end{aligned}
$$

Theorem 2 implies that

$$
M_{p} \circ M_{q} \sim \begin{cases}M_{r} & r=\max \{p, q\}, p \neq q \\ M_{L^{q} \log L} & p=q .\end{cases}
$$

Let us note that in some very special cases one can determine the constants $c_{1}, c_{2}$.

Example 2. For $r>1$, if $f$ is a nonincreasing function $f:(0, \infty) \rightarrow$ $(0, \infty)$ then

$$
M_{r} f(x) \leq M\left(M_{r} f(x)\right) \leq \frac{r}{r-1} M_{r} f(x),
$$


by Kolmogorov inequality (see [BDS]). Moreover

$$
\begin{aligned}
f_{0}^{x} f(t)\left[1+\log \frac{x f(t)}{\int_{0}^{x} f(s) d s}\right] d t & \leq M(M f(x)) \\
& \leq \frac{e}{e-1} f_{0}^{x} f(t)\left[1+\log \frac{x f(t)}{\int_{0}^{x} f(s) d s}\right] d t
\end{aligned}
$$

Let us consider Bagby's formula, (see [B]), for such $f$. If $\Phi_{\alpha}(t)=t[1+$ $\left.\left(\log ^{+} t\right)^{\alpha}\right], \alpha>0$, then

$$
c_{1} M_{\Phi_{\alpha}} f(t) \leq \frac{1}{t} \int_{0}^{t}\left(\log \frac{t}{s}\right)^{\alpha} f(s) d s \leq c_{2} M_{\Phi_{\alpha}} f(t),
$$

and observe that $M_{\Phi_{\alpha}} \circ M_{\Phi_{\beta}} \sim M_{\Phi_{\alpha+\beta+1}}$.

Remark 2. If in Theorem $2 \Phi(t)=t$, we get

$$
\frac{\Theta(t)}{t}=\int_{0}^{t} \frac{\Psi^{\prime}(s)}{s} d s .
$$

It is easy to verify that

$$
c_{1} M_{\Psi} f(x) \leq M_{\Psi}(M f)(x) \leq c_{2} M_{\Psi} f(x)
$$

if and only if $\Psi(t)=A(t) t^{p}$, where $A(t)$ is a continuous increasing function and $p>1$.

Moreover, we have that

$$
c_{1} M_{\Phi} f(x) \leq M\left(M_{\Phi} f\right)(x) \leq c_{2} M_{\Phi} f(x)
$$

if and only if $\Phi(t)=t^{p}$, where $p>1$. So, we reobtain the mentioned result of $[\mathbf{C R}]$.

\section{Some consequences.}

Corollary 1. Let $A(t)$ be a Young function in $(0, \infty)$. The n-composition, $M_{A} \circ M_{A} \circ \cdots \circ M_{A}$, of the maximal operator $M_{A}$, is equivalent to the maximal operator $M_{A_{n}}$, where

$$
A_{n}(t)=\int_{0}^{t} A^{\prime}(s) A_{n-1}\left(\frac{t}{s}\right) d s .
$$


Proof: Put $A_{1}(t)=A(t)$. For $n=2$, (3.1) follows by Theorem 1 . By an induction argument, we have the assertion for every positive integer $n$.

In particular, the $n$-iterate of the Hardy-Littlewood maximal operator is equivalent to the operator

(see $[\mathbf{L}],[\mathbf{L N}],[\mathbf{P}])$.

$$
M_{L \log ^{n-1} L}
$$

Let $f$ be a measurable function defined on $\mathbb{R}^{n}$. We denote by $\mu$ the distribution function of $f$, namely, for $t>0$ we set

$$
\mu(t)=\left|\left\{x \in \mathbb{R}^{n}:|f(x)|>t\right\}\right| .
$$

Then we define the decreasing rearrangement $f^{*}$ of $f$ :

$$
f^{*}(s)=\sup \{t>0: \mu(t)>s\} \quad(s>0) .
$$

The following theorem states the equivalence between $(M f)^{*}$ and $M\left(f^{*}\right)$.

Theorem 3 (Herz). If $f \in L_{\text {loc }}^{1}\left(\mathbb{R}^{n}\right)$ then $\forall t>0$ it holds that

$$
4^{-n}(M f)^{*}(t) \leq M\left(f^{*}\right)(t) \leq\left(2^{n}+1\right)(M f)^{*}(t) .
$$

\section{Proof: See $[\mathbf{B S}]$.}

Another corollary of Theorem 2 is the following Herz type inequality for the $L \log ^{n} L$-maximal operator.

Corollary 2. There exist $c_{1}(n), c_{2}(n)>0$ such that, if $f$ belongs to $L \log ^{k} L, k \in \mathbb{N}$, then $\forall t>0$

$$
c_{1}\left(M_{L \log ^{k} L} f\right)^{*}(t) \leq M_{L \log ^{k} L}\left(f^{*}\right)(t) \leq c_{2}\left(M_{L \log ^{k} L} f\right)^{*}(t) .
$$

Proof: Let us prove the thesis for $k=1$. Herz inequality states that

$$
c_{1}(M g)^{*}(t) \leq M\left(g^{*}\right)(t) \leq c_{2}(M g)^{*}(t)
$$

Formula (3.2), with $g$ replaced by $M f$, becomes

$$
c_{1}(M \circ M(f))^{*}(t) \leq M\left((M f)^{*}\right)(t) \leq c_{2}(M \circ M(f))^{*}(t)
$$

and using (3.2) again in the right hand side of (3.3), we get

$$
(M \circ M(f))^{*}(t) \sim M\left((M f)^{*}\right)(t) \sim M \circ M\left(f^{*}\right) .
$$

Applying Theorem 1 in (3.4), with $\Phi(t)=\Psi(t)=t$, we get

$$
\left(M_{L \log L} f\right)^{*}(t) \sim(M \circ M(f))^{*} \sim M_{L \log L}\left(f^{*}\right)(t) .
$$

The thesis follows arguing by induction.

As another application of Theorem 2, we obtain a pointwise estimate about the maximal function of the jacobian of a function $f$ (see [IS], $[\mathbf{M}])$. Namely we have the following 
Theorem 4. If $|D f|^{n} \in L_{\text {loc }}^{1}\left(\mathbb{R}^{n}\right)$, then we have

$$
M_{L \log L} J(x) \leq c(n) M\left(|D f|^{n}\right)(x) \quad \text { a.e. } \quad x \in \mathbb{R}^{n}
$$

where $J=J_{f}(x) \geq 0$ is the jacobian of $f$.

Proof: In [IS] is proved that if $|D f|^{n} \in L_{\text {loc }}^{1}\left(\mathbb{R}^{n}\right)$ for any cube $Q \subset \mathbb{R}^{n}$, $0<\sigma<1$, then we have

$$
f_{\sigma Q} J d y \leq c(n)\left[f_{Q}|D f|^{\frac{n^{2}}{n+1}} d y\right]^{\frac{n+1}{n}},
$$

and so

$$
M J(x) \leq c(n)\left[M\left(|D f|^{n \frac{n}{n+1}}\right)\right]^{\frac{n+1}{n}}(x) .
$$

Now, thanks to Theorem 2, applying the maximal function $M$ to both sides in (3.7), we get

$$
\begin{aligned}
M_{L \log L}(J)(x) & \leq c M\left[\left(M\left(|D f|^{\frac{n}{n+1}}\right)\right)^{\frac{n+1}{n}}\right](x) \\
& =c\left(M_{\frac{n+1}{n}}\left(M\left[|D f|^{\frac{n^{2}}{n+1}}\right]\right)(x)\right)^{\frac{n+1}{n}} \\
& \leq c M\left(|D f|^{n}\right)
\end{aligned}
$$

as desired.

Remark 3. Let us observe that, in general, the following estimate

$$
M_{\Theta} J(x) \leq c(n) M_{\Psi}\left(|D f|^{n}\right)(x) \quad \text { a.e. } \quad x \in \mathbb{R}^{n}
$$

holds, where $\Psi(t)$ is a Young function, $|D f|^{n} \in L_{\mathrm{loc}}^{\Psi}\left(\mathbb{R}^{n}\right)$ and

$$
\Theta(t)=\Psi(t)+\frac{1}{n+1} t \int_{0}^{t} \frac{\Psi(s)}{s^{2}} d s .
$$

\section{References}

[B] R. J. BAGBy, Maximal functions and rearrangements: some new proofs, Indiana Univ. Math. J. 32(6) (1983), 879-891.

[BDS] L. Basile, L. D'Apuzzo and M. Squillante, Relation between Gehring and Muckenhoupt classes via the Hardy operator, Ricerche Mat., (to appear). 
[BP] R. J. Bagby and J. D. Parsons, Orlicz spaces and rearranged maximal functions, Math. Nachr., 132 (1987), 15-27.

[BS] C. Bennet and R. Sharpley, "Interpolation of Operators," Academic Press, 1988.

[CR] R. R. CoIfman And R. Rochberg, Another characterization of BMO, Proc. Amer. Math. Soc. 79 (1980), 249-254.

[G] L. Greco, A remark on the equality $\operatorname{det} D f=\operatorname{Det} D f$, Differential Integral Equations 6(5) (1993), 1089-1100.

[GIM] L. Greco, T. Iwaniec and G. Moscariello, Limits of the improved integrability of the volume forms, Indiana Univ. Math. J., (to appear).

[IS] T. Iwaniec And C. Sbordone, On the integrability of the Jacobian under minimal hypothesis, Arch. Rational Mech. Anal. 119 (1992), 129-143.

[KR] M. A. Kranosel'skit and Y. B. Rutickit, "Convex functions and Orlicz spaces," Noordhoff, Groningen, 1961.

[L] M. A. Leckband, A note on maximal operators and reversible weak type inequalities, Proc. Amer. Math. Soc. 92 (1984), 19-26.

[LN] M. A. Leckband and C. J. Neugebauer, A general maximal operator and the $A_{p}$ condition, Trans. Amer. Math. Soc. 275 (1983), 821-831.

[M1] G. Moscariello, A pointwise inequality for Riesz Potentials in Orlicz spaces, Rend. Accad. Sci. Fis. Mat. Napoli(4) 53 (1986), $41-47$.

[M2] G. Moscariello, On the integrability of the jacobian in Orlicz spaces, Math. Japon. 2 (1994), 323-329.

[Mu] S. Muller, Higher integrability of determinants and weak convergence in $L^{1}, J$. Reine Angew. Math. 412 (1990), 20-34.

[P] C. PÉREZ, Endpoint estimates for commutators of singular integral operators, (to appear).

[RR] M. M. RaO and Z. D. Ren, "Theory of Orlicz spaces," Marcel Dekker, 1991.

[S] E. M. Stein, Note on the class L $\log L$, Studia Math. 32 (1969), 305-310. 
[T] A. Torchinsky, "Real variable methods in harmonic analysis," Academic Press, 1987.

Keywords. Maximal function, Orlicz spaces.

Dipartimento di Matematica e Applicazioni "R. Caccioppoli" Universita'degli studi di Napoli Federico II

Via Cintia

80126 Napoli

ITALY

Primera versió rebuda el 19 de Desembre de 1995,

darrera versió rebuda el 28 de Maig de 1996 